\title{
Smart Fridges with Multimedia Capability for Better Nutrition and Health
}

\author{
Suhuai Luo, Hongfeng Xia, Yuan Gao, Jesse S. Jin, and Rukshan Athauda \\ School of Design, Communication \& Information Technology \\ The University of Newcastle, Callaghan NSW 2308 \\ suhuai.luo@newcastle.edu.au
}

\begin{abstract}
Due to the development of computer technology and the wide use of the Internet, intelligent appliances with multimedia capability have been emerging into our daily life. Kitchen is one of the places where such intelligent appliances have been used. Since modern life style is driving people spending less time on cooking healthy food at home, an enjoyable and healthy life style can be assisted with an intelligent kitchenware such as a smart fridge. In this paper we introduce a novel application for a smart fridge with intelligent multimedia capability. It is designed for managing items stored in it and advising its users with cooking methods depending on what kind of food is stored. It can also perform other functions such as dietary control, nutrition monitoring, eating habit analysis, etc. We are confident that such a smart fridge will be an important component in future smart homes.
\end{abstract}

\section{Introduction}

Both industry and research have focused on developing smart homes [1-4]. For example, more than 100 homes offering smart technology have been built in South Korea and another 30,000 are planned [5]. Research has also focused on developing smart homes for the elderly [6]. We have seen and will continue to see significant strides in this area which will make a major impact on how we life.

Developing smart appliances is critical to the realization of a smart home [7]. Kitchen is a prominent place in the home consisting of many intelligent appliances which aim to provide better services for a household. A smart fridge is the focus of our research and we see many efforts in developing the smart fridge in industry and research. The industry attempts to change the traditional fridge function which stores food items in a cool environment to integrating fridge with $\mathrm{TV}$, radio, and computer capabilities and even connection to the Internet. These computer-like capabilities enable the development of applications for devices such as smart fridges. In this project, we aim to develop an application for a smart fridge that focuses on better health and nutrition.

It is a well-known fact that the fast-paced development and modern living has resulted in people lifestyle with less exercise and an unhealthy diet [8]. Poor eating patterns can cause diet related illness in late life if we do not take eating nutritional food seriously [9]. For instance, obesity resulting from unhealthy dietary habits and lifestyles is becoming a major health hazard in the developed countries. In this context, we believe that applications which focus on better nutrition and health are important. This paper presents an application geared towards the smart fridge which promotes better nutritional and health habits. The fridge being a prominent appliance in the kitchen plays an important role in food and nutrition of a household and in our opinion is ideally suited for such an application.

\section{Conceptual smart fridge models}

In this section, we describe some of the emerging smart fridge concepts and features contributed by different manufacturers.

\subsection{Siemens CoolMedia Fridge Freezer}

Siemens CoolMedia Fridge Freezer KG39MT90 [10] has built in 15" LCD TV and comes with remote control and cable or DVD sockets. The difference between this LCD TV and other TV is that the screen can be swiveled out. So you can cook and watch Siemens CoolMedia TV from practically anywhere in the kitchen. The fridge aims to encourage eating and watching $\mathrm{TV}$ in the kitchen areas rather than in the living room. .

\subsection{LG TV Refrigerator}


The newest LG TV Refrigerator LSC27990TT [11] has remote-controlled, cable-ready 15" LCD TV with DVD connection and FM radio, also has 4" Weather Plus LCD display with forecasts based on users' area, date/time, calendar with alarm, eight-category recipe bank with 100 preloaded recipes and personal digital photo album. Other features include child lock function, room temperature display, digital user manual and product controls.

\subsection{Electrolux Screen Fridge}

The Electrolux Screen Fridge [12] has the function of broadband connection and TV via wireless connection. As if Internet, email, phone, radio and MP3 player are not enough, not only comes with the $15^{\prime \prime}$ touch screen and pop-up keyboard, but also Electrolux adds highly advanced calendar and video messaging system so the kitchen truly becomes the center hub in the house. Every morning, users can check weather and traffic conditions, business appointments with Screen Fridge personal organizer, before you leave, and also easily print out updatable electronic shopping list. The refrigerator is also accessible by remote access feature.

\subsection{Samsung Smart Zipel Refrigerator}

If you're the one who can't be without TV or connectivity to the net even for a second, Samsung has its Smart Zipel refrigerator [13] for you that wears a 10.4-inch wireless LCD screen on its chest like a badge of honour. The $4 \times 3$ flat panel display lets you connect to anything remotely multimedia such as video or the Web. The Internet access might be convenient if you need to display a recipe you've found on the Web while you're busy preparing it in the kitchen. Details are sketchy at this point with no pricing or availability information forthcoming, but the refrigerator looks like a fairly highend unit, capable of holding 26.6 cubic feet of groceries.

\subsection{The main shortage of current smart fridge and nutrition issue}

- Technology is too complex for most household users, needing more friendly interface for normal user (no computer use experiences).

- No function of diet or nutrition

- No meal organizer and healthy food suggestion function

- No uniform of bar code (include expire date)

- Limited by the network connection(speed and support)
- Potential privacy issues. Because the networked home would enable information to flow out of the home in ways that households are not accustomed to, privacy could be compromised.

- Requiring a new, unique operating system for remote device use to control the smart system

- Different producers develop different products

\section{Fridge with nutritional functionality}

The fridges made with advanced technology are more concentrated on the combination of intelligent sensor $[14,15]$, network and information. We can see trends in developing the fridge to act as a computer, TV, DVD player etc., however, applications focused on healthy nutritional habits and lifestyles is lacking. We see an opportunity and worthwhile to develop such applications for Smart Fridges. This paper presents the development of such an application. We believe that applications focusing on nutritional information and dietary habits of its users to be a valuable application for a Smart Fridge as it is an integral part of the kitchen. Healthy nutrition intake can have far reaching and significant impact on health and well-being of consumers.

\subsection{Perspective of the project}

The main aim of the project is the development of a Smart Fridge application focusing on nutrition facts and dietary habits of its users. The application has a database which contains specific nutrition information stored inside its central chip. The application maintains information regarding each specific user such as weight, height, age, medical record, allergic food to name a few. It scans every item while the item passes the door sensor so that the fridge can record the type, the quantity and the expiry date of that item. At the time when the date is getting close to the expiring date of an item stored in the fridge, it will notify the user either by video or audio warning sign. In addition, information is displayed on the screen mounted on the door of the fridge to enquire if the user wants to use it or not. With respect to different personal preferences, the fridge is able to list the essential shopping list for that particular person once a week referring to the items which are running out.

\subsection{The unique features - nutritional recipes}

A significant feature in this application is that the fridge is intelligent to propose suitable recipes for different types of people according to the materials 
stored in it. It will create more than one recipe that can be chosen from using different cooking methods or seasoning. Further, if a user has pre-determined illness (such as high-blood pressure, diabetes etc.), the fridge will notify the user with the food nutrition facts and indicate what kind of food is appropriate.

\subsection{Functions and features of smart fridge}

Following are a list features implemented in the Smart Fridge application

- Generating recipe suggestions based on food stored in the fridge

- Multimedia cooking demonstration (needs connectivitiy to Internet)

- Generating store list

- Generating shopping list

- Warning on food which is going to expire

- Displaying calories for various foods

- Scanning food and storing its information into a database

- Friendly control panel

- $\quad$ Storing family information and medical record.

- Calculating BMI (Body Mass Index)

- Nutrition suggestion based on BMI and for various user with illnesses such as diabetes, high blood pressure

\section{System design and implementation}

\subsection{System implementation}

For this smart fridge application, we used Microsoft SQL Server 2005 to build the database and Microsoft Visual Studio 2005 to create the user interface.

The system follows a two-tier architectural design. User interacts with the interface of the smart fridge. VB.NET code connects to the database using SQL Server classes. All the functions like insertion, modification and deletion are manipulated using stored procedures.

\subsection{Database Design}

There are 15 tables in the database which are described below:

Home Member: This table represents the all user information, including user name, DOB, height, weight, gender etc.

Medical_Recorder: In this table, there are MR_ID and Detail columns. They store the information about every user's medical recorder, and are connected to
Home_Member table by MR_ID primary and foreign key connection.

Nutrient: This table includes all possible nutrients in each dish and item.

Nutrient_Taken_Limitation: This table contains the data about different nutrient limitations for different diseases.

Bar_code: The columns in this table include barcode, product name, units, quantity, expire date etc. It stores all different products' information.

Bar_Nutrient: This table connects Bar_Code and nutrient tables, representing how much different nutrition each product contains.

Stored_Item: In this table, the columns include

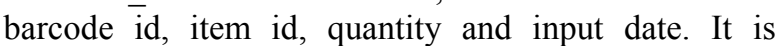
connected to Barcode by barcode id P/F key connection.

Course: This table contains course id, name, CT_ID, method, demo and picture and so on. All courses user can choose are stored in this table.

Course_Type: This table stores the information about the main course section and connects with course by CT_ID.

Course Nutrient: This is the middle table between course and nutrient table. The columns include: Course_ID, Nutrient_ID, Units and quantity.

Course_Order: This is connection table between Course and Home Member table. This table stores the information about different user ordered different course and order date too.

Ingredient: The columns in this table are Ingredient_ID, name, units. This table stores the information about all ingredients used in cooking different dishes.

Flavouring: The columns in this table are Flavouring_ID, name, units. This table stores the information about all flavourings using in cooking different dishes.

Course_Ingredient: This is the middle table connecting the course table and Ingredient table and represent each course use how many ingredients. The columns include Course_ID, Ingredient_ID, Units and quantity.

Course_Flavouring: This is the middle table connecting the course table and Flavouring table and represents each course use how many flavouring. The columns include Course_ID, Flavouring_ID, Units and quantity.

The relational schema is presented in Figure 1.

\subsection{Interface Design}

The control panel of the smart fridge receives the requests made by user and displays the results back to user (Figure 2). 
The recipes screen displays recipes in two different ways. One is to create recipes based on food stored in the fridge, and another is to generate recipes from database based on different types of dishes the user selects (Figure 3).

The stored list is to display various foods stored in the fridge on the touch screen of fridge without opening the door of fridge. It also contains information about food name, expiry date, weight (Figure 4).

The cooking demo shows the cooking demonstration video of each course. It helps users to feel more realistic when they are cooking (Figure 5).

The smart fridge is able to store information of each member in a family with their medical records so that it can create suitable dishes for those who have diseases like diabetes or high blood pressure (Figure $6)$.

The BMI Calculator allows users to check if they are normal or overweight based on their height and weight (Figure 7).

The nutrients check is to show the nutrients of each course and the nutrition facts of each item by entering its barcode (Figure 8).

The shopping list screen is to display a suggested shopping list to the user so that the user needs not to bother to know what to buy (Figure 9)

\section{Conclusion and future work}

We have introduced a novel smart fridge application with intelligent multimedia capability. Although the proposed model has shown its potential in maintaining better nutrition and health, it is still in its preliminary stage. There are many features and areas that can be improved and explored. Possible extensions include:

- Internet connectivity: allowing downloading of recipes in real time, online shopping instruction etc.

- RFID tag scanning system.

- Inside fridge steelyard function. A scale integrated inside the fridge allow automatically determine usage for food or drink such as milk.

- Weekly meal design, including previous meal nutrient calculation and meal design.

- Using microwave system to defrost frozen food automatically before use

- Connecting to oven, controlling the time of oven when baking

- Mobile control: Integrating functionality to interface with the fridge using the mobile phone.

\section{Acknowledgement}

We would like to thank Dr. Justin Singleton for his comments on nutrient study, calculation of the BMR and BMI, and other helpful suggestion

\section{References}

[1] R. Beuran, J. Nakata, T. Okada, T. Miyachi, K. Chinen, Y. Tan, Y. Shinoda (2007), Performance Assessment of Ubiquitous Networked Systems, 5th International Conference on Smart Homes and Health Telematics (ICOST2007), Nara, Japan, June 21-23, 2007, pp. 19-26.

[2] Laura Cerwinske (2004), Smart Home Design, Reed Press

[3] ElecVision (2006), Smart Home Solutions, www.elecvision.com.au

[4] Robert C.Elsenpeter, Toby J.Velte (2003), Find in a Library: Build your own smart home, McGraw-Hill

[5] Dan Simmons (2006), Smart homes a reality in S Korea, http://news.bbc.co.uk/2/hi/programmes/click_online/617 9868.stm.

[6] Dana Christianson (2005), Home smart home, http://www.researchworks.carleton.ca/2005_Spring/79.h tm

[7] Jiang,L. and Yang,B. (2004), Smart Home Research, Proceedings of the IEEE International Conference on Machine Learning and Cybernetics, Shanghai, 26-29 August 2004

[8] Catherine E.Woteki and Paul R.Thomas (1992), Eat For Life: The Food and Nutrition Board's Guide to Reducing Your Risk of Chronic Disease Washington, D.C. National Academies Press.

[9] Nicola King (2003), Smart Home-What do consumers want?, Intertek Research\&Test Centre

[10] http://www.appliancist.com/refrigerators/siemenscoolmedia-fridge-freezer.html

[11] http://www.appliancist.com/refrigerators/lg-tvrefrigerator-lsc27990tt.html

[12] http://www.appliancist.com/refrigerators/electroluxscreen-fridge.html

[13] http:/gizmodo.com/gadgets/homeentertainment/samsung-smart-zipel-refrigerator156257.php

[14] White, N. M., et al. (2007), Sensors in Adaptronics, Springer Berlin Heidelberg, ISBN, 978-3-540-71965-6.

[15] Li, J., Guo, Y., and Poulton, G. (2004), Critical Damage Reporting in Intelligent Sensor Networks, Proceedings, 17th Australian Joint Conference on Artificial Intelligence - AI 2004: Advances in Artificial Intelligence, 6th - 10th December 2004, Cairns, Australia, vol. 3339, pp. 26-38. 


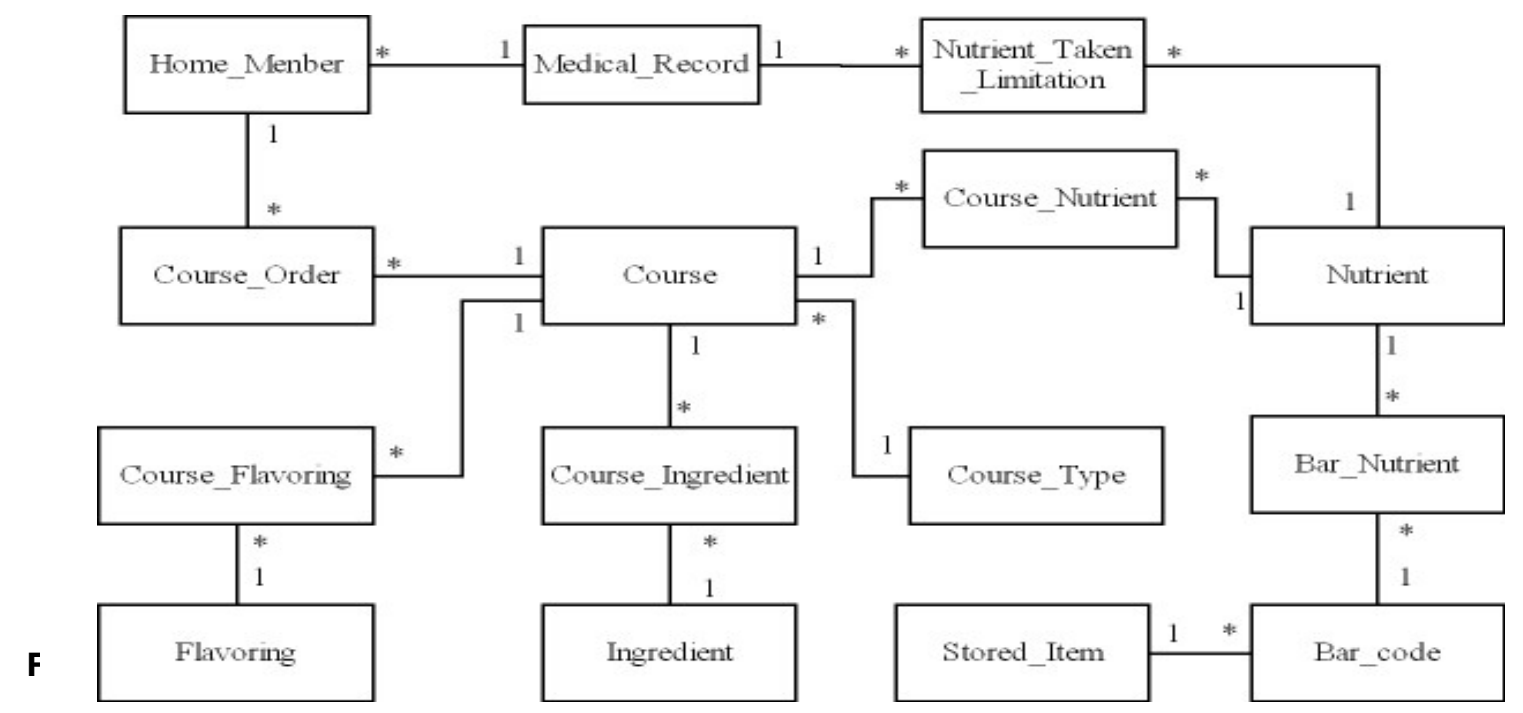

igure.1 Smart Fridge E-R diagram. Where square block indicates entity; connecting line indicates relationships between entities; 1 indicates single relationship; * indicates multiple relationship.

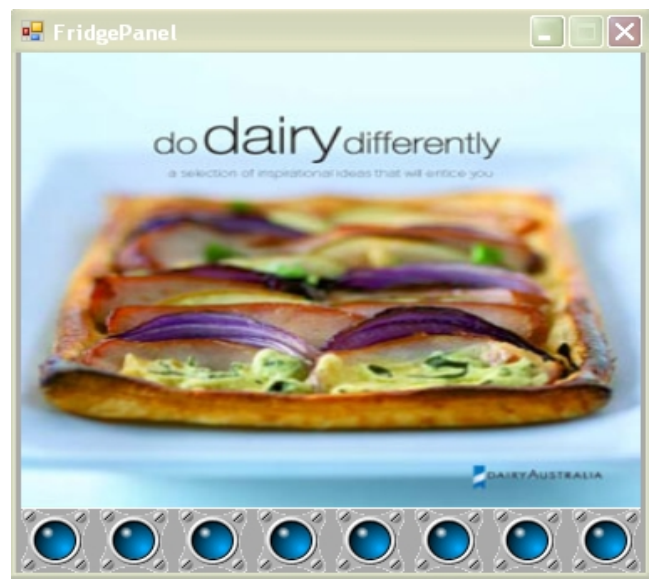

Figure 2 Fridge Control Panel

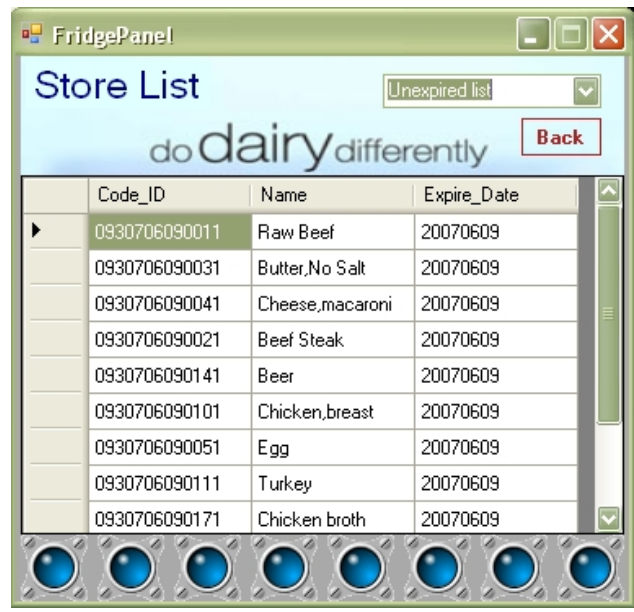

Figure 4 Store List Panel

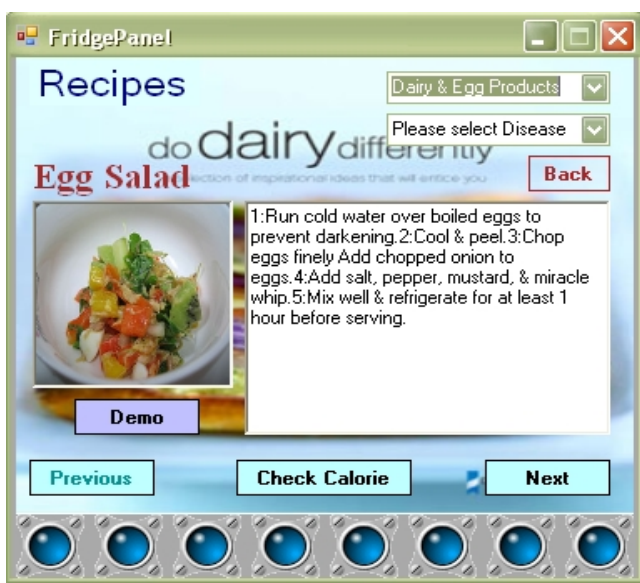

Figure 3 Recipes Panel

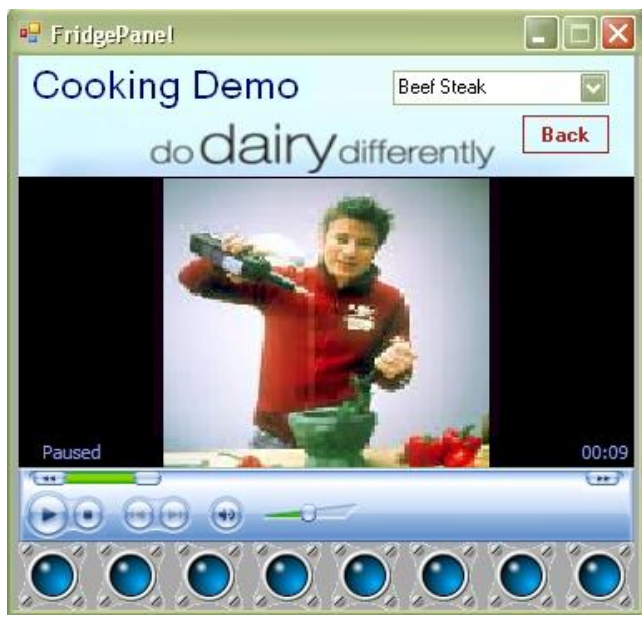

Figure 5 Cooking Demo Panel 


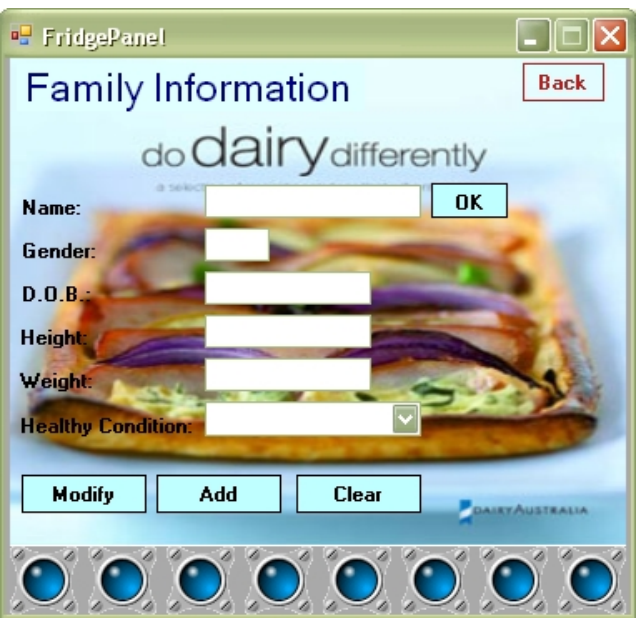

Figure 6 Family Information Panel

\begin{tabular}{|c|c|c|c|c|}
\hline \multicolumn{4}{|c|}{ 口 FridgePanel } & $=\square x$ \\
\hline \multicolumn{4}{|c|}{ Nutrients Check } & Back \\
\hline \multicolumn{5}{|c|}{ Bar Code: diun y Check antly } \\
\hline & Course_Name & Nutrient & Quantity & Units \\
\hline \multirow[t]{7}{*}{ • } & Egg Salad & Energy & 191 & c \\
\hline & Egg Salad & Protein & 13.3 & g \\
\hline & Egg Salad & Carbohydrate & 3.8 & $g$ \\
\hline & Egg Salad & Sugar & 11.2 & $g$ \\
\hline & Egg Salad & Fat & 13.5 & $g$ \\
\hline & Egg Salad & Vitamin & 82.5 & $\mathrm{mg}$ \\
\hline & Egg Salad & Cholesterol & 427 & $\mathrm{mg}$ \\
\hline * & & & & \\
\hline$<$ & & IIIII & & 7 \\
\hline
\end{tabular}

Figure 8 Nutrients Check Panel

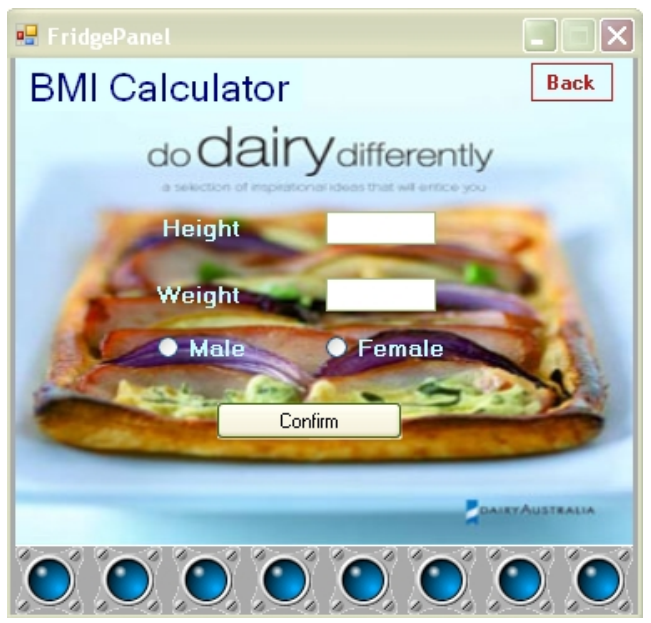

Figure 7 BMI Calculator Panel

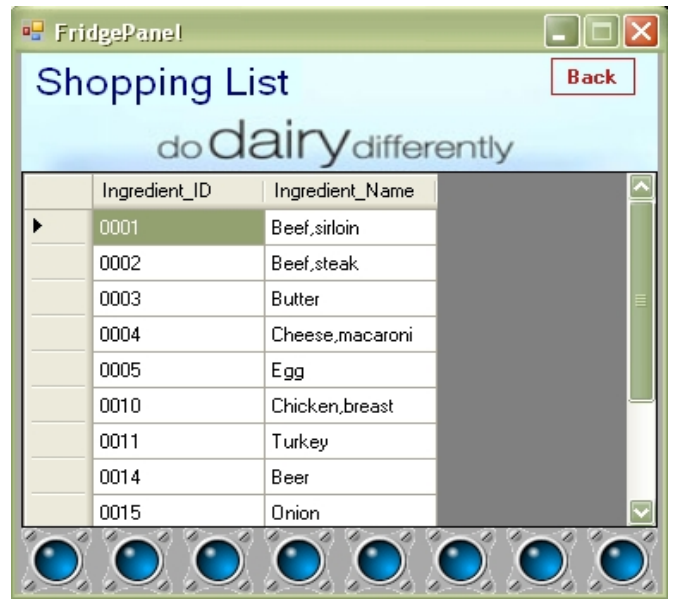

Figure 9 Shopping list Panel 